Journal of Mathematics and Statistics 3 (4): 163-167, 2007

ISSN 1549-3644

(C) 2007 Science Publications

\title{
Mathematical Sophistication and Differentiated Emotions during Mathematical Problem Solving
}

\author{
${ }^{1}$ Bradford D. Allen and ${ }^{2}$ James Carifio \\ ${ }^{1}$ Lasell College, ${ }^{2}$ University of Massachusetts-Lowell
}

\begin{abstract}
George Polya, author of Mathematical Discovery and the enduring best seller How to Solve It: A New Aspect of Mathematical Method, claims that more sophisticated people experience more differentiated emotion during mathematical problem solving, a claim that has been untested for nearly fifty years. Using instruments that measure ongoing evaluations and emotion in real time, and traits that reflect mathematical sophistication, the effect of sophistication on emotion during mathematical problem solving was investigated in this study using 209 mathematics and science majors at a technological university in the northeast. Students who were more mathematically sophisticated, as defined by Polya, were better problem solvers and had more highly differentiated emotions and ongoing self-evaluations of progress towards solutions than students where were not mathematically sophisticated, just as Polya claimed. These more sophisticated students also managed their negative emotions and anxiety during problem solving better than students who were not mathematically sophisticated. It is hypothesized that these sophisticated students would be good mentors or work partners for students who were less mathematically sophisticated as they would model these metacognitive skills and traits for these later students helping them to learn them.
\end{abstract}

Key Words: Emotions, Mathematical Problem Solving, Polya, Mathematical Sophistication, MetaCognition, Dynamic Assessment.

\section{INTRODUCTION}

The importance of emotion during mathematical problem solving has long been recognized ${ }^{[1,}{ }^{2}$. Emotion can organize, focus, disrupt, distract or energize problem solving ${ }^{[3]}$, and the influence of emotion can be immediate or delayed ${ }^{[4]}$. Emotion influences representational aspects of problem solving [5], and emotion forms enduring affective pathways that contribute to an individual's mathematical powers ${ }^{[6]}$. In his classic book How to Solve It, Polya ${ }^{[7]}$ claims that if the student had no opportunity in school to familiarize himself with the varying emotions of the struggle for the solution, his mathematical education failed him in the most vital of ways.

In important work, according to Schoenfeld ${ }^{[8]}$, on the cognitive activities that occur during mathematical problem solving, Polya ${ }^{[9,7]}$ describes the ongoing cognitive evaluations that lead to emotion ${ }^{[10,11]}$ for full details on these points. In particular, Polya ${ }^{[7]}$ claims that more sophisticated people experience more differentiated emotion during problem solving. Polya does not define sophistication, but in the context of problem solving, Polya is most probably referring to individuals who have greater experience and expertise in problem solving, who perform better at problem solving, and who have positive attitudes toward mathematics ${ }^{[9]}$. This definition of sophistication is similar to Bloom's ${ }^{[12]}$ definition of sophistication, and Polya was familiar with Bloom's work. Thus, for this study, sophistication can be defined and measured in terms of affect and problem-solving performance.

Three instruments developed by the authors were used to measure evaluations, affective traits, emotion, and problem solving expertise, and sophistication. The Emotion Questionnaire ${ }^{[13]}$ is a 32 item semantic differential constructed using Gable and Wolf's [14] approach to instrument development over the affective domain. Emotion components, which include Polya's evaluations, were measured by five to eight item subscales. The subscales and their alpha internal consistency estimates of reliability are the following: perceived physiology, $\alpha=.84$; positive and negative emotion, $\alpha=.84$; relevancy, $\alpha=.84$; proximity, $\alpha=.93$; and quality, $\alpha=.96$. These alpha coefficients are excellent given that the subscales contain only 5 to 8 items each. The validity of the subscales was established by factor analysis.

The Mathematics Affect Trait Questionnaire ${ }^{[15]}$ was used to measure individual differences in traits that influence learning, testing, and affect as identified by Anderson ${ }^{[16]}$. Anderson's five affective traits are 
academic motivation, academic self-esteem, mathematics anxiety, interests in mathematics, and locus of control. Each of the traits was measured by a 5item subscale with items purposefully selected from validated tests recommended by Anderson. Response formats were changed from 4 and 5 point Likert scales to a 6 point Likert scale to increase item variance for better correlation and effects estimates. The alpha internal consistency estimates of reliability for the 5item subscales are academic motivation $\alpha=.27$; academic self-esteem $\alpha=.52$; mathematics anxiety $\alpha=54$; interests in school $\alpha=80$; and locus of control $\alpha$ $=29$. The alpha coefficients are somewhat low, even for subscales with only 5 items, because the subscales contained high unique-variance items designed to obtain heterogeneous profiles of the traits being measured. Moreover, each subscale is measuring a trait that is only weakly correlated with the other four traits and, therefore, is a concurrently valid measure.

This point and fact can be seen from the communality estimates of the proportion of variance shared with other factors which are academic motivation, $\mathrm{h} 2=.35$; academic self-esteem, $\mathrm{h} 2=.32$; mathematics anxiety, $\mathrm{h} 2=.38$; interests in school, $\mathrm{h} 2=.59$; and locus of control, h2 =.19.

The Math Problem Set consisted of one easy problem and one difficult problem. The two "Polya problems" ${ }^{[10]}$ were randomly selected from a validated set of twenty-four problems. One problem was a traditional algebra word problem. The second problem was a novel, unconventional problem designed to challenge students' problem solving skills. The contrast between these two problems are discussed by Polya in his work, but is also similar to the "novice-expert contrast" used in modern cognitive psychology.

In other work, Carifio and Allen ${ }^{[11]}$ map and translate Polya's theories and views of problems, problem solving and the problem solving processes into fairly standard modern views of cognitive psychology ${ }^{[18]}$ and emotion ${ }^{[19]}$. However, this earlier work did not articulate nor test Polya's views and hypotheses about the role and function of sophistication in problem solving, which is the focus of this work.

For this study, two-hundred-nine students from a public university in the northeast with strong scientific and technological programs participated in a study to investigate the effect of mathematical sophistication on emotion during problem solving,. The sample size was selected to be consistent with Everitt's ${ }^{[20]}$ and Kass and Tinsley's ${ }^{[21]}$ guidelines for factor stability.
The subjects in the study met in a classroom in groups of 25 for one hour. The experimental procedures were read and then the subjects spent fifteen minutes completing the Mathematics Affect Trait Questionnaire. The subjects then spent thirty-five minutes trying to solve two math problems in random order. To sample moment-to-moment changes in evaluation and emotion, the subjects completed six semantic differential Emotion Questionnaires while trying to solve the two problems. Each questionnaire took less than two minutes to complete. In all, 152 students worked on the two problems and completed all items on the Math Affect Trait Questionnaire and six Emotion Questionnaires.

Solutions to the two problems were assigned correctness scores by the first-named author using a holistic scoring technique devised by the California State Department of Education Assessment Program. This rubric scores each answer as either an exemplary response, 6 points; competent response, 5 points; satisfactory with minor flaws, 4 points; nearly satisfactory but contains serious flaws, 3 points; begins problem but fails to complete solution, 2 points; fails to begin effectively, 1 point; or no attempt at solution, 0 points.

Sophistication: To test Polya's claim, sophistication was operationalized in terms of affect and problem solving performance. Anderson's five affective traits (described above) reflect sophistication because of their ability to predict knowing, learning, and problem solving performance. Factor analysis, correlation analysis, and stepwise multiple regression results all show that the affective traits that best predict problem correctness scores and emotion were math interest, selfesteem, and lack of anxiety.

With an increase from one extreme -- low mathinterest, low academic-self-esteem, and high mathematics-anxiety (typical of low sophistication), to the opposite extreme -- high math-interest, high academic-self-esteem, and low mathematics-anxiety (typical of high sophistication), evaluations of. relevancy, proximity, and quality; emotion; and problem correctness scores all increased. Because math interest, self-esteem, and lack of anxiety, are good predictors of affect, emotion, knowing, learning, and problem correctness, and because performance is an important aspect of Polya's view of sophistication, then sophistication was operationalized as the sum of the problem correctness scores, math interest, academic self-esteem, and the absence of mathematics anxiety. 
Emotion: Polya's claim about differentiated emotion seems to imply that it takes more independent and differentiated emotion constructs to describe the emotion of sophisticated problem solvers than it does to describe the emotion of unsophisticated problem solvers. To test whether more independent (and thus, differentiated) emotion constructs (i.e., factors) are required to explain the emotion of sophisticated problem solvers than would be required to explain the emotion of unsophisticated problem solvers, the subjects were ranked according to their level of sophistication and then divided into low, medium, and high sophistication groups. When the emotion responses of each group were factor analyzed, the results showed that the same number of emotion constructs was required to explain most of the emotion variance for the high sophistication and low sophistication students alike. That is, the dimensions of self-reported emotion for the high and low sophistication groups were statistically the same.

At this point, the following question arose: Were our results inconsistent with Polya's claim, or was our operational definition of emotion too narrow? Given the semantic differential format that was used for measuring real-time self-reported emotions, and the manner in which it was scored, we adopted the view that true emotion arises from changes in progress/performance evaluations (as Polya claims) and thus, from changes in reported real-time emotions. This viewpoint and dynamic is similar to Mandler's [19,22] view that emotion arises from changes in evaluations due to the interruption of plans or expectations.

Differentiated Emotion: It was evident from the beginning that many students reported uniformly positive or uniformly negative emotion on one or more semantic differential variables while solving a given problem. Other students reported volatile emotion that jumped from positive-to-negative, or round-trip positive-to-negative-to-positive (or the reverse) on one or more variables. Therefore, to further investigate Polya's claim that more sophisticated people experience more differentiated emotion during problem solving, the variation in evaluations and self- reported emotion was used as a measure of true emotion. Further, the numbers of independent self-reported emotion variation constructs, that is, the dimensionalities of true emotion, were investigated. Polya's claim would be supported if more independent constructs (factors) are required to describe self-reported emotion variation (true emotion) for the high sophistication problem solvers.
To investigate the dimensions of emotion variation across 32 evaluation and emotion variables for the high and low sophistication students, the variation was found for each emotion variable using the responses on the three Emotion Questionnaire surveys that were completed for the two problems. That is, all the student's responses were used to compute a variation measure for each variable. This averaging process effectively reduced the number of data records from 912 to $(912) /(3 * 2)=152$ records. Because factor stability was a concern, a reduction in the number of emotion variation variables was necessary. The variation measures of the 32 semantic differential variables were reduced to six true emotion scales by factoring the 32 emotion variation measures into six principle components. The four highest loading variation variables for each component were averaged to get six true emotion scales. Following this data reduction, 152 records with six scales each were ranked according to their sophistication level and then divided into low, medium, and high sophistication groups. The six scales were then factor analyzed for the high and low sophistication groups separately.

\section{FACTOR ANALYTICAL RESULTS}

Factor analyses of the scales were conducted using the principal-components factor method with communalities assumed equal to one (not to be confused with simply finding the principal components of the correlation matrix). The analysis showed that emotion variation of high sophistication students has a higher dimensionality than for low sophistication students. Specifically, a greater number of independent constructs (factors) was required to account for most of the variance of the high sophistication students. For example, to account for at least 80 percent of the variance in true emotion, only one emotion construct was required for the low sophistication group, but two true emotion constructs were required for the high sophistication group. The second construct had an eigenvalue equal to .74 , but a scree test on the eigenvalues indicated that this second factor should be retained. To account for at least 90 percent of the variance in true emotion, only two constructs were required for the low sophistication group, but four constructs were required for the high sophistication group. In this comparison however, the second and forth factors of the low and high sophistication groups respectively, had eigenvalues equal to .43 so these factors may represent a significant amount of measurement error. However, when all the factors with eigenvalues less than one in magnitude were removed, 
only one factor was retained for both the low and high sophistication groups but the unique variance for the six scales of the high sophistications group was one to FIVE times as large as for the low sophistication group.

\section{DISCUSSION}

The analyses that were conducted showed that selfreported emotion variation (true emotion) for high sophistication students contains more independent and differentiated constructs. The significantly greater number of emotion constructs (factors) required to explain true emotion for the high sophistication group, as compared to the low sophistication group, and the significantly greater unique variance for the high sophistication scales indicates that more sophisticated students experience more differentiated emotion during problem solving.

Differences between the specific constructs of the high sophistication and low sophistication groups were evident. In the case where the constructs accounted for 90 percent of the variance, the variation of true emotion of the low sophistication group was explained by

(1) the perceived quality of the student's work, and

(2) self-reported physiological evaluations.

On the other hand, the emotion of high sophistication students was explained by

(1) the quality of the students' work,

(2) how relevant the problem was to the students,

(3) how close the students were to the solution, and

(4) how certain/uncertain the students were in their work.

Moreover, for the low sophistication group, feeling smart or dumb was independent of other evaluations and emotion; similarly, feeling quick or slow in solving the problem was independent of other evaluations and emotion. For the high sophistication group, feeling smart or dumb was correlated with the level of confidence about how to solve the problem, and being quick or slow was correlated with feeling smart or dumb. For the high sophistication group, emotion was more comprehensive in the way it was broadly based on many evaluations. Changes in evaluations and emotion for high sophistication students were correlated with many more semantic differential variables than was the case for the low sophistication students. Changes in emotion for the low sophistication group occurred along single semantic differential variables and were independent of other evaluation and emotion variables. In other words, progress/performance evaluations and the emotions of low sophistication students had "hot spots" that were activated by performance and ability.
Though both groups reported high and low extremes on all semantic differential evaluation variables and emotion variation scales, on average, high sophistication problem solvers had emotion scores that were about one standard deviation more positive than the emotion scores of their low sophistication counterparts.

It is commonly believed that the main effects of emotion in problem solving are disruptive and distracting and that negative emotion interferes with and diminishes performance. The empirical evidence presented here, however, does not support these beliefs. Instead, these results indicate that emotion energizes, organizes, focuses, and improves performance. Furthermore, emotion provide highly valuable problem solving information for sophisticated problem solvers.

The results of this study have immediate implications for the widespread "feel good" approaches used to teach mathematics during the last decade. In particular, these teaching approaches were misdirected in their over-generalized and undifferentiated views about emotion and its role in problem solving and learning ${ }^{[24-26]}$. Cognitive dissonance and emotional conflict, within appropriate limits, have positive psychological and learning functions and are not inimical factors to be eliminated from the learning process. Allowing students to have both positive and negative emotion as an integral part of mathematics learning is neither demeaning nor detrimental. Instead, according to these results, a varied-emotion approach generates valuable meta-cognitive evaluation information as well as stimulating, energizing, organizing, and focusing effects from the concomitant emotion.

A key to success in problem solving, according to the results presented here, occurs when students consider the cognitive evaluations generated during problem solving to be personally important and then when they constructively utilize the differentiated emotions that occur as a result. If the importance of cognitive evaluations and concomitant emotion is taught as part of a general model of problem solving practice, the use of authentic, challenging, and intrinsically relevant problems, where students have a personal stake in the outcome, may be particularly beneficial in helping students become better problem solvers. As Polya claims, more sophisticated people experience more differentiated emotion during problem solving.

Lastly, the results indicate that less sophisticated problem solvers would gain valuable problem solving 
experience from having more sophisticated problem solvers act as role models and peer mentors in cooperative learning situations. This mentoring approach might be most effective, from a long-term benefit perspective, when applied in middle school contexts where students encounter elementary but real problems for the first time. Mentoring may help less sophisticated problems solvers learn to attend to the four components of problem solving that generate emotion in sophisticated problem solvers, learn to control and to some degree ignore much of the physiological components of emotion that occurs during mathematical problem solving, have more balanced physiological emotion regulation, and become better and more sophisticated mathematical problem solvers.

\section{REFERENCES}

1. Dreger, R. M., \& Aiken, L. R. 1957. The Identification of Number Anxiety in a College Population. Journal of Educational Psychology, 10, 344-351.

2. Higbee, J. L., \& Thomas, P. V. 1999. Affective and Cognitive Factors Related to Mathematics Achievement. Journal of Developmental Education, 23(1), 8-32.

3. Abella, R., \& Heslin, R. 1989. Appraisal Processes, Coping and the Regulation of Stress-Related Emotion in a College Examination. Basic and Applied Social Psychology,_10(4), 311-327.

4. Lazarus, R. S. 1991 Emotion and Adaptation. New York: Oxford University Press.

5. DeBellis, V. A., \& Goldin, G. A. 1997. The Affective Domain in Mathematical Problem Solving. In E. Pehkonen (Ed.) Proceedings of the 21th Annual Meeting of PME-NA: Vol. 2, 209216.

6. Goldin, G. A. 2000. Affective Pathways and Representation in Mathematical Problem Solving. Mathematics Thinking and Learning, 2(3), 209219.

7. Polya, G. 1985. How to Solve It: A New Aspect of Mathematical Method. New Jersey: Princeton University Press.

8. Schoenfeld, A. H. 2000. George Polya and Mathematics Education. In G. Alexanderson's The Random Walks of George Polya. The Mathematical Association of America.

9. Polya, G. 1981. Mathematical Discovery. New York: Wiley.

10. Allen, B. D. and Carifio, J. 1995. The Relationship Between Emotional States and Solving Complex Mathematical Problems. Columbus, OH: Educational Resources Information Center (No. ED 392 605).
11. Allen, B. D. \& Carifio, J. 2004. Mathematical Discovery: A Covariance Analysis. Academic Exchange Quarterly, 8(2), 115-119.

12. Bloom, B. S. 1956. Taxonomy of Educational Objectives: The Classification of Educational Goals, Handbook I: Cognitive Domain. New York: David McKay Company.

13. Allen, B. D. \& Carifio, J. 1999a. The Development and Validation of an Emotion Questionnaire for the Investigation of Affect During Mathematical Problem Solving. Springfield, VA: ERIC Document Reproduction Service No. ED434037.

14. Carifio, J. and Allen, B. 2006. A Set of Polya Problems for Studying Mathematical Problem Solving. Paper presented at the Annual Conference of the Eastern Education Research Association, Hilton Head, S.C.

15. Allen, B. D. \& Carifio, J. 1999b. The Development and Validation of a Math Affect Trait Questionnaire for the Investigation of Affect During Mathematical Problem Solving. Springfield, VA: ERIC Document Reproduction Service No. ED434038.

16. Anderson, L. W. 1981. Assessing affective characteristics in schools. Boston, Allyn \& Bacon.

17. Allen, B. D. \& Carifio, J. 1999c. A Problem Set for the Investigation of Mathematical Problem Solving. Springfield, VA: ERIC Document Reproduction Service No. ED434039.

18. Carifio, J. 2005 An integrated information processing theory of learning. Proceedings of the Eighth International History, Philosophy \& Science Teaching Conference, Leeds, England, p 100-172. Available

at: http://www.ihpst2005.leeds.ac.uk/papers/Carifio.pdf

19. Mandler, G. 1975. Mind and Emotion. New York: John Wiley.

20. Everitt, B. S. 1975. Multivariate analysis: The need for data, and other problems. British Journal of Psychiatry, 126, 237-240.

21. Kass, R. A. \& Tinsley, H. E. 1979. Factor analysis. Journal of Leisure Research, 11, 120-138.

22. Mandler, G. 1989. Affect and Learning: Causes and Consequences of Emotional Interaction. In D. B. McLeod \& V. M. Adams (Eds), Affect and Mathematical Problem Solving: A New Perspective. NY: Springer-Verlag.

23. Poincaré, H. 1952. Science and method. New York: Dover Publications, Inc.

24. Hadamard, J. 1954. The psychology of invention in the mathematical field. New York, NY: Dover Publications.

25. McLeod, D. B. \& Adams, V. M. 1989. Affect and mathematical problem solving: A new perspective. NY: Springer-Verlag.

26. Zan, R.; Brown, L.; Evans, J.; \& Hannula, M. 2006. Affect in Mathematics Education: An Introduction. Educational Studies in Mathematics, 63(2), pp. 113-121. 